\title{
Du commerce équitable au commerce éthique : principes et enjeux d'une extension des règles de justice sociale
}

\section{Anne Joyeau et Philippe Robert-Demontrond}

\section{QpenEdition}

\section{Journals}

Édition électronique

URL : http://journals.openedition.org/communicationorganisation/3273

DOI : 10.4000/communicationorganisation.3273

ISSN : 1775-3546

Éditeur

Presses universitaires de Bordeaux

Édition imprimée

Date de publication : 2 janvier 2005

Pagination : $56-67$

ISSN : 1168-5549

\section{Référence électronique}

Anne Joyeau et Philippe Robert-Demontrond, « Du commerce équitable au commerce éthique : principes et enjeux d'une extension des règles de justice sociale », Communication et organisation [En ligne], 26 | 2005, mis en ligne le 19 juin 2012, consulté le 30 avril 2019. URL : http:// journals.openedition.org/communicationorganisation/3273; DOI : 10.4000/ communicationorganisation.3273

Ce document a été généré automatiquement le 30 avril 2019

(C) Presses universitaires de Bordeaux 


\title{
Du commerce équitable au commerce éthique : principes et enjeux d'une extension des règles de justice sociale
}

\author{
Anne Joyeau et Philippe Robert-Demontrond
}

1 Si pour certains, la distinction entre commerce équitable et commerce éthique devient caduque (Soenens, 2002), c'est essentiellement parce que ces deux démarches poursuivent le même objectif : rééquilibrer et rendre plus transparentes les relations commerciales entre les producteurs ou salariés des pays en voie de développement et les consommateurs des pays industrialisés. Et cependant, on assiste actuellement au développement d'initiatives de plus en plus formalisées, par le biais de la labellisation ou de la certification, relevant soit du commerce dit «équitable » soit, plus récemment, du commerce dit "éthique ». Après avoir exposé ces deux formes de commerce « alternatives ", à la fois sous l'angle de leurs principes et de leur dynamique, les limites opérationnelles des pratiques de labellisation sociale seront soulignées.

\section{Principes du commerce équitable}

\section{Caractéristiques du commerce équitable}

2 Visant à établir un rapport d'échanges satisfaisants pour tous, le commerce équitable est fondé sur cinq principaux objectifs: 1) assurer une rémunération du travail des producteurs leur permettant de répondre à leurs besoins élémentaires; 2) refuser systématiquement une quelconque forme d'esclavage ou de travail forcé, y compris l'exploitation des enfants; 3) instaurer des relations durables entre partenaires économiques; 4) favoriser la préservation de l'environnement; 5) proposer aux consommateurs des produits de qualité. Et ce, à partir d'une dénonciation des mécanismes actuels des échanges commerciaux internationaux, opérant audétriment des producteurs et des consommateurs. 
3 Pour préserver leur dignité et les préserver de la pauvreté, les organisations du commerce équitable garantissent aux producteurs un prix minimum et des avances de paiement et surtout, la plus grande transparence possible sur la formation du prix des produits, en rendant publique la part revenant à chaque opérateur. Les circuits de distribution étant volontairement plus courts que ceux traditionnels, limitant donc le plus possible les intermédiaires, le surcoût à l'achat ne se retrouve que pour partie dans le prix finalement payé par les consommateurs motivés par des principes de solidarité.

4 En cela donc, le commerce équitable s'inscrit typiquement dans le programme de l'Agenda 21 (programme d'actions pour le XXI siècle défini à Rio, en 1992, lors de la conférence des Nations-Unies sur l'environnement et le développement), qui pose très explicitement que le développement durable peut être accéléré par une coopération internationale basée sur « un système commercial multilatéral ouvert, équitable, sûr, non discriminatoire, prévisible, compatible avec les objectifs du développement durable, et conduisant à une répartition optimale de la production mondiale selon les principes de l'avantage comparatif ${ }^{1}$.

\section{Les initiatives du commerce équitable}

Reposant sur un système de magasins spécialisés et de labels, un marché « équitable » s'est progressivement développé : ainsi par exemple, l'association française « Artisans du Monde ", créée dès 1974 dans le prolongement des idées lancées en 1968 contre les mécanismes de subvention financière - trade, not aid -, qui travaille à l'instauration d'échanges commerciaux équitables. Encore marginale en France, la part de ce commerce est cependant croissante, notamment grâce à la diffusion récente, dans des circuits de distribution classiques tels que la grande distribution, de produits certifiés pour lesquels un label garantit qu'un prix équitable est payé à leur producteur. Ainsi « Max Havelaar », association néerlandaise créée en 1988, qui propose un label international garantissant aux consommateurs des produits équitables ${ }^{2}$.

6 Pour l'essentiel, les diverses organisations françaises engagées dans le développement du commerce équitable sont réunies dans un organisme de représentation national, créé en 1997 : la Plate-forme pour le Commerce Équitable. Sa principale mission, outre la veille à ce que personne ne puisse se prévaloir de faire du commerce équitable s'il n'y adhère pas, est la promotion et la consolidation du commerce équitable, la coordination des actions entreprises. Son action aboutit à la répartition des marchés nationaux européens entre trois labels de commerce équitable : Fair Trade Mark, pour l'Irlande et le Royaume-Uni ; Max Havelaar, pour les Pays-Bas, la Belgique, la France, le Danemark et la Suisse ; Transfair, pour l'Autriche, l'Allemagne, l'Italie et le Luxembourg. Labels qui sont accessibles dans les quelques 3000 "Magasins du monde» du réseau, également chez des détaillants classiques, spécialistes - Artisans du Monde, Oxfam, Fair Trade, Human Inside, Boutic Ethic, Max Havelaar, Alter Eco - ou généralistes, avec l'irruption de la grande distribution sur ce créneau de marché.

\section{Dynamique du commerce équitable}

7 Si les premières expériences du commerce équitable datent à présent de près de 40 ans, apparaissant sous l'expression de "commerce alternatif » aux débuts des années 60 au Royaume-Uni et aux Pays-Bas, son essor, comme alternative viable aux circuits de 
distribution conventionnels est très récent, conditionné par le développement d'une demande solvable. Sa dynamique est ainsi très étroitement liée à sa professionnalisation et à l'engagement de campagnes destinées à responsabiliser les consommateurs du Nord à générer le marché...

Surtout, d'importantes évolutions conceptuelles se font actuellement jour, affectant le contenu du commerce équitable.

Alors que celui-ci était initialement pensé, essentiellement, dans une perspective de rapports Nord-Sud focalisée sur les exportations comme vecteur de développement économique, et considérant que l'amélioration des conditions tarifaires des exportations devait mécaniquement favoriser le développement des pays du Sud, les questions liées à la finitude des ressources physiques, d'une part, et aux limites économiques du marché mondial, d'autre part, sont de plus en plus prises en considération. Le projet «labels régionaux » de Yamana et l'initiative du Comercio Justo pour la promotion du commerce équitable à l'intérieur du Mexique sont ainsi particulièrement suivis, s'agissant de systématiser les méthodologies appliquées pour évaluer l'opportunité de les appliquer en d'autres régions du monde - y compris en France. Le concept de «commercialisation communautaire", militant pour la limitation des flux et le développement de formes d'échanges internes au Sud et au Nord, rencontre ainsi dès à présent un vif intérêt en Amérique Centrale et Amérique du Sud ${ }^{3}$.

Par ailleurs, après avoir impliqué les matières premières et les produits manufacturés, le commerce équitable s'étend à présent au secteur tertiaire. Actuellement, le tourisme est perçu comme étant loin d'être un vecteur évident de développement durable, mais apparait plutôt, souvent, comme un facteur d'inégalités et de déséquilibres, écologiques et socio-économiques. De là, la construction de projets visant à en faire un support de développement local, permettant aux populations de s'approprier la maitrise des projets touristiques et de recevoir une part significative des bénéfices ${ }^{4}$. Ce tourisme, équitable, pensé dans le prolongement des expériences du tourisme rural et du tourisme social, doit servir de catalyseur à l'innovation sociale et territoriale et à la conservation des patrimoines naturels et culturels.

11 Enfin, le commerce équitable présente aujourd'hui des affinités d'importance avec d'autres initiatives visant également au développement durable : ainsi notamment des programmes d'écologie industrielle et de l'agriculture biologique. Si ce mouvement prête attention au respect des conditions sociales de production, les produits du commerce équitable sont très souvent, pour ceux alimentaires, de qualité biologique. Autant d'éléments qui brouillent les frontières... Notamment du point de vue du consommateur, pouvant être plus sensible, lors de l'achat d'un produit labellisé « équitable », à sa qualité supposée qu'à ses conditions de production ou aux modalités de rémunération de ses producteurs.

12 Mais c'est aussi le champ d'action de certaines organisations qui travaillaient au départ au développement du commerce équitable qui s'étend progressivement, s'orientant vers les conditions de travail des salariés produisant les biens: les labels originairement consacrés à la promotion du commerce équitable, tel le label Max Havelaar, se rapprochent ainsi aujourd'hui des initiatives relevant du commerce éthique. 


\section{Principes du commerce éthique : une labellisation sociale élargie}

\section{Caractéristiques du commerce éthique}

Relativement au commerce équitable, le commerce éthique s'intéresse plus particulièrement au cas où les producteurs à soutenir sont des salariés, tout en reposant sur des objectifs plus étendus - visant de fait non seulement à offrir des conditions de travail et de rémunération du travail décentes, mais également à assurer le respect de la liberté syndicale, l'absence de discrimination à l'embauche et au travail, etc. Le commerce éthique met ainsi explicitement l'accent sur la responsabilité sociale des entreprises.

\section{Les initiatives du commerce éthique}

14 Trois formes d'initiatives, finalisées par le développement du commerce éthique, peuvent être distinguées : i) les actions volontaires d'entreprises, aboutissant à la construction de chartes sociales ou de codes de conduite internes ou propres à un secteur d'activité donné ${ }^{5}$ et inventoriant des référentiels sociaux ; ii) les actions menées par des organismes nonmarchands pour la sensibilisation des consommateurs aux enjeux du commerce éthique, aboutissant à la création de labels sociaux apposés sur un produit; iii) les actions d'organismes indépendants visant à certifier les entreprises qui respectent des normes sociales dites « standards »-- de type SA 8000.

15 1) Texte réunissant les engagements qu'une entreprise s'oblige à respecter en matière de conditions sociales de production, un code de conduite définit les limites dans lesquelles une entreprise considère devoir assumer sa responsabilité en matière sociale. De plus en plus d'entreprises présentes dans le commerce international, transnationales notamment, tendent à s'imposer volontairement de telles restrictions, souvent sous la pression d'ONG très actives et par voie de suite, des consommateurs.

2) Conçus par des organismes indépendants, les labels sociaux offrent quant à eux la garantie que les produits les arborant ont été fabriqués en respectant les normes de travail retenues par l'organisme en question ${ }^{6}$. Dans son livre vert, la Commission des Communautés européennes définit le label social comme des « mots ou symboles apposés sur un produit dont le but est d'influer sur la décision d'achat des consommateurs en apportant une garantie sur l'impact social et éthique d'un processus commercial sur les autres parties prenantes concernées ".. ${ }^{7}$. A cet effet, le label social doit présenter deux qualités fondamentales: i) la fiabilité, afin de permettre aux consommateurs de faire aisément la différence entre les produits respectant les critères sociaux et ceux qui supposés ne pas les respecter; ii) l'efficacité, ce qui implique d'être immédiatement reconnaissable à l'intérieur des points de vente.

3)Enfin, sur le modèle des normes ISO 9000 et ISO 14000 respectivement destinées à contrôler la qualité des produits et les pratiques environnementales, des organismes de certification sociale ont vu le jour, visant à contrôler la responsabilité sociale d'une entreprise (et de ses fournisseurs, sous-traitants éventuels) dans ses pratiques commerciales. Ainsi le SAI (Social Accountability International), anciennement CEPAA (Council on Economic Priorities Accreditation Agency) qui a mis en place, en octobre 1997, la norme sociale standard internationale SA 8000, et le système d'accréditation qui 
l'accompagne. Exclusivement basée sur les résolutions de l'OIT, la norme SA 8000 fait l'objet d'un processus régulier de réévaluation : une première révision a déjà eu lieu, et c'est aujourd'hui la norme SA 8000 version 2002 qui sert de référence. Une cinquantaine d'entreprises au monde sont actuellement certifiées SA 8000.

\section{Limites opérationnelles de la labellisation sociale ${ }^{8}$}

18 Le principal risque qu'encourt actuellement le commerce éthique tient à son actuelle récupération stratégique. Les thèmes de responsabilité sociale, d'éthique, de solidarité transnationale, de soutenabilité environnementale et sociale du développement économique, sont à la mode et peuvent être, dans des marchés saturés, tant pour les producteurs que pour les distributeurs, d'excellents supports de différenciation des offres commerciales. Et ce, sans conditionalité d'adhésion effective aux règles: ce qui, évidemment, pose le problème de possibles dérives affectant, sur le long terme, l'image de ce marché et donc sa viabilité.

\section{Problèmes liés à la définition des engagements}

19 Les problèmes liés à la définition des engagements, tiennent à ce que les engagements comportementaux au fondement des pratiques de labellisation sociale :

- 1)se situent souvent très en deçà des normes internationales applicables en matière de conditions de travail - et ce, en ne traitant qu'un nombre limité de droits, par conséquent choisis ;

- 2)sont éventuellement pénalisants pour les entreprises adoptant des codes significativement plus contraignants que leurs compétiteurs, dans des régimes stratégiques de domination par les coûts ;

- 3)s'appliquent souvent à des entreprises de sous-traitance ayant plusieurs donneurs d'ordre

- les chartes ou codes de conduite pouvant alors définir des contraintes hétérogènes ;

- 4)ne contraignent pas les entreprises à divulguer des informations sur leurs activités ;

- 5)ne contiennent souvent pas de clauses relatives à une vérification indépendante.

S'agissant du premier point, les codes de conduite sont unilatéralement produits par les entreprises et sont fondés sur leur volontariat. Volontariat qui, lui-même, relève significativement plus du calcul stratégique, d'une logique économique de rapports coûts / bénéfices - que d'un raisonnement déontologique kantien. En conséquence, peu des codes écrits reprennent dans leurs engagements publics les droits sociaux fondamentaux reconnus par l'OIT. La plupart ne retiennent souvent guère que les principaux thèmes auxquels le public est sensible: travail des enfants, discrimination raciale, sexuelle, etc.

21 Concernant le second point, les labels sociaux sont souvent appréhendés par les firmes comme des instruments de différenciation de l'offre commerciale permettant de construire un positionnement attractif, également valorisant en terme de communication institutionnelle, et autorisant une montée en gamme, en termes de prix. De là l'engouement des entreprises transnationales pour le commerce éthique. Ce qui pose alors problème en ce que les possibilités de certification (très coûteuses) et de communication sur la certification ne sont pas également réparties entre toutes les entreprises : les PME risquent de subir une forte distorsion de concurrence. 

de rapports publics ; 2) la définition de mesures correctives en cas de non-respect ; 3) des normes relatives à la divulgation publique d'informations sur les conditions de travail. Entre autres exemples, la FSE-THC (Textile, Habillement et Cuir), en coopération avec l'Union Européenne (la DG Emploi et affaires sociales), débuta en 1992 un programme visant à la création d'une charte sociale - aboutissant le 22 septembre 1997 à la signature d'un accord avec l'organisation européenne des industries textile-habillement. Mais, pour autant qu'un tel accord ait été diffusé à plus de $40 \%$ des entreprises européennes du secteur, et intégré dans les conventions collectives de six des pays de l'Union, les comportements des inspecteurs du travail n'ont pas été significativement modifiés - des enfants de moins de 15 ans continuant à travailler en nombre dans plusieurs des pays concernés.

\section{Problèmes liés à la vérification des engagements}

Si l'octroi d'un label social implique que l'ensemble de la chaîne de production remplisse les critères exigés, l'idée que chaque entreprise ayant participé au processus de production soit certifiée pour que le produit final obtienne un label garantissant le respect des normes sociales est d'applicabilité très limitée.

Plus généralement, l'information même sur le respect effectif des engagements pris, peut être émise par : 1) les entreprises elles-mêmes, qui assurent la vérification auprès de leurs sous-traitants ; 2) des organismes indépendants d'évaluation et de certification qui renseignent consommateurs et investisseurs individuels des modes de production des entreprises ; 3) des organismes indépendants, des associations et organisations non gouvernementales, assurant la vérification des engagements et organisant une pression continue sur les entreprises pour qu'elles les respectent effectivement; 4) des médias, tout particulièrement des revues et des publications des associations de consommateurs et des mouvements consuméristes; 5) des organisations gouvernementales et supragouvernementales, finalement, comme l'OIT.

26 Si différentes solutions s'offrent ainsi afin de vérifier les engagements pris par les entreprises en matière de responsabilité sociale, c'est aujourd'hui essentiellement à ce niveau que les initiatives dites « éthiques » se révèlent défaillantes. Or, le contrôle est un aspect essentiel de la labellisation sociale : la publication, par une entreprise, d'un code de conduite ne suffit en effet pas à assurer son application, notamment dans des pays géographiquement lointains où les intermédiaires sont souvent nombreux et où la législation locale peut s'avérer imprécise. Même dans le cas de la norme SA 8000, vis-à-vis de laquelle les ONG françaises ne semblent pas hostiles, le rôle insuffisant joué par les ONG locales dans le processus de certification pose problème et reste le point faible de ce système (Benchetritt, 2000). Quel que soit le système mis en place (code de conduite, charte sociale, label, norme), les pratiques d'entreprises sont vivement critiquées sur la question du mode de contrôle et actuellement, aucun système ne semble idéal. 


\section{Conclusion}

$27 \mathrm{Au}$ plan opérationnel, les limites des initiatives qui s'inscrivent dans le cadre du commerce éthique, synthétisées dans le tableau 1, sont donc aujourd'hui considérables. Dans le domaine de labellisation sociale, un travail important reste donc à faire afin de garantir le sens éthique, et pas uniquement commercial, des actions menées. Brand et Hoffmann (1994) citent à ce titre l'exemple de pratiques de labellisation qui pourraient ouvrir des possibilités d'accès à l'emploi pour les chômeurs, ceci grâce au recul du travail des enfants, de manière à empêcher une dégradation supplémentaire du niveau de vie dans les pays concernés. Mais surtout, le défi à relever aujourd'hui est celui de la consolidation des réseaux, de la mise en place de structures durables : la multiplicité des actions et des acteurs, que ce soit dans le commerce dit « équitable » ou « éthique », qui, si elle traduit une progression de ces secteurs, peut aussi freiner leur développement. L'information donnée aux consommateurs restant à ce jour incomplète, manquant parfois de fiabilité, d'impartialité (Kapstein, 2001), les risques sont élevés que le consommateur ressente une certaine méfiance à l'égard de ce nouveau marché : seule une information claire permettra de faire en sorte que la qualité sociale des produits devienne un réel critère de choix lors de l'achat, au-delà des seules déclarations d'intentions.

Tableau 1 - Les initiatives du commerce éthique

\begin{tabular}{|c|c|c|c|}
\hline & Contenu/Objet & Finalite/Objectif & Limites \\
\hline $\begin{array}{l}\text { Chartes } \\
\text { sociales } \\
\text { codes de } \\
\text { conduite } \\
\text { Labels } \\
\text { sociaux } \\
\text { Normes } \\
\text { sociales }\end{array}$ & $\begin{array}{l}\text { Garantie de certains critères } \\
\text { sociaux lors de la fabrication } \\
\text { d'un produit (entreprise ou } \\
\text { secteur d'activités) } \\
\text { Labels de qualité sociale d'un } \\
\text { produit, de certification des } \\
\text { méthodes de production } \\
\text { Norme standard, "idéale " } \\
\text { (de type SA 8000) certifiant la } \\
\text { responsabilté sociale d'une } \\
\text { entreprise }\end{array}$ & $\begin{array}{l}\text { Au minimum, } \\
\text { couverture des } \\
\text { conventions } \\
\text { fondamentales de l'OIT } \\
\text { Sensibilisation du } \\
\text { consommateur et donc, } \\
\text { des distributeurs } \\
\text { Application universelle } \\
\text { de standards sociaux }\end{array}$ & 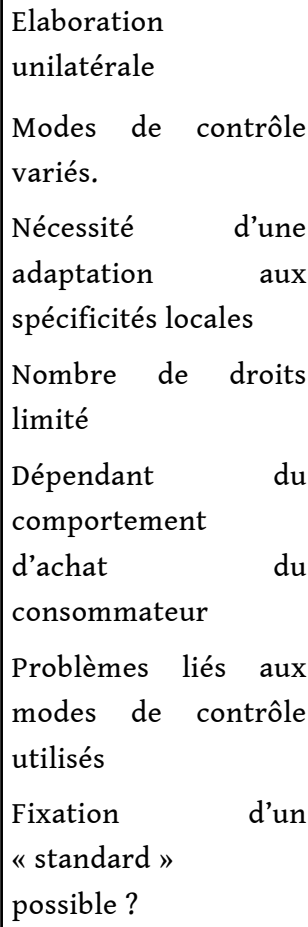 \\
\hline
\end{tabular}

La définition de principes communs entre les diverses parties prenantes du commerce équitable et éthique se révèle donc indispensable pour assurer une meilleure lisibilité des pratiques. C'est dans cette perspective qu'une réflexion est en cours dans plusieurs pays 
européens, visant à la régulation du commerce éthique (Chosson, Johnson, 2002). En France, un groupe de travail mis en place par l'AFNOR (Association Française de Normalisation) a pour mission de réfléchir à cette question. Par ailleurs, si ces critiques d'ordre opérationnel adressables à l'encontre des actuelles politiques de labellisation sociale s'avèrent nombreuses, d'autres problèmes d'ordre conceptuel se posent encore ${ }^{9}$, tenant notamment à ce que les codes de conduite et autres labels sociaux s'appuient à présent tous, directement ou indirectement, explicitement ou implicitement, sur l'idée qu'existent des valeurs universelles. La tâche reste donc immense pour instaurer de nouvelles règles aux échanges mondiaux.

\section{BIBLIOGRAPHIE}

«La Consommation citoyenne » (2003), Dossier Alternatives Economiques, Hors Série, $\mathrm{n}^{\circ} 10$, Mars.

Benchetritt N. (2000), « Label social. Polyesther 80\%, Ethique 20\% », Alternatives Economiques, juillet.

Brand D., Hoffmann R. (1994), « Le débat sur l'introduction d'une clause sociale dans le système commercial international : quels enjeux ? ", Problèmes Economiques, $\mathrm{n}^{\circ} 2.400,30$ novembre.

Canel-Depitre B. (2001), «L'entreprise face aux engagements du consommateur-citoyen » Revue Française de Gestion, $\mathrm{n}^{\circ} 136$, novembre-décembre.

Chosson A., Johnson P. (2002), « Commerce éthique et équitable », Rapport des groupes de travail du Comité français pour le sommet mondial du développement durable.

Kapstein E.B. (2001), «L'éthique à tout prix ? ", Problèmes Economiques, n².739, 5 décembre.

Peeters A. (2001), «Codes de conduite et labels sociaux : le meilleur et le prix », Courriel d'information ATTAC n² 295.

Robert-Demontrond P., Basset G. (2004), « La labellisation sociale de l'offre commerciale : potentialités et limites ", Gestion 2000, mai-Juin.

Soenens R. (sous la Direction de), Soumeryn-Schmit O., Constantinescu D. (2002), « Responsabilité sociale. Manuel pour détaillants en textile », Projet de l'Association Européenne des organisations nationales des Détaillants en Textile (AEDT).

Somavia J. (2001), « Pour l'instauration de règles du jeu équitable », Le Monde Diplomatique, septembre, p.26.

\section{NOTES}

1. Extrait de l'Agenda 21, section 1, chapitre 2.

2. Premier label international concernant l'alimentation (café, thé, banane, jus d'orange, miel et riz), Max Havelaar est présent dans 17 pays et représente 800000 producteurs du Sud. 
3. Cf. les résultats de la «Rencontre latino-américaine sur le commerce équitable et la consommation éthique dans la perspective de la solidarité globale», tenue du 15 au 17 mars 2001 à Lima - URL : fairtrade.socioeco.org/documents/

4. A ce titre, un collectif «Tourisme équitable» est présent au sein de la Plate Forme pour le Commerce Equitable évoquée plus avant.

5. Ainsi le code «CCC » de la Clean Clothes Campaign, réseau d'ONG travaillant sur les questions du commerce éthique dans le secteur des vêtements au sens large du terme.

6. Les critères retenus sont le plus souvent édités dans un code de conduite ou peuvent correspondre à l'application d'une norme sociale standard: ainsi par exemple le label LINK, directement apposé sur le produit, octroyé par une société suisse, AMANA SA, et basé sur le respect de la norme SA 8000.

7. Cf. Commission de l'Union européenne, Livre vert, Promouvoir un cadre européen pour la responsabilité sociale des entreprises, juillet 2001.

8. La labellisation sociale est ici entendue au sens large, comprenant l'ensemble des initiatives du commerce éthique : labels, codes de conduite ou chartes sociales, normes sociales.

9. Sur ce thème, cf. l'article de Robert-Demontrond $\mathrm{P}$. publié dans ce même numéro de Communication et Organisation.

\section{RÉSUMÉS}

C'est dans un contexte d'inquiétude croissante face à la mondialisation que les consommateurs, depuis récemment, manifestent des attentes en matière d'éthique économique et ce, dans une perspective de développement soutenable, postulant qu'un développement à long terme ne peut être viable qu'en parvenant à intégrer à la fois la rentabilité économique, le respect de l'environnement et l'équité sociale. Les entreprises se trouvent ainsi incitées à réfléchir à une labellisation de leur offre intégrant des promesses de plus en plus larges de justice : au-delà du commerce équitable développé dans les années 60 , se met ainsi progressivement en place un commerce dit "éthique » à travers des pratiques diverses de labellisation sociale - expression regroupant ici les chartes sociales, les codes de conduite, les labels sociaux ou encore les normes sociales -, plaçant la responsabilité sociale des entreprises au centre du dispositif. L'objet de cet article est d'étudier la dynamique de ce mouvement en présentant ces deux formes de commerce sous l'angle de leurs principes et de leurs enjeux, mais surtout, en exposant les limites opérationnelles dont la labellisation sociale fait l'objet.

In a context of globalization of the economy, and in a sustainable development logic, consumers are today waiting for the integration of ethic criteria in the economy. Entreprises try to bring some answers by developping systems of certification of their products, with more end more large promises of justice. Beyond the "fair trade", appeared in the 60's, a form of "ethic trade" is now developed, through different tools of social certification (social charts, codes of conduct, social labels or social norms), putting the social responsability of enterprises in the center of the system. The object of this article is to study the dynamics of this evolution. These two forms of trade will be presented : their principles, their stakes and, above all, their limits will be developed. 


\section{AUTEURS}

\section{ANNE JOYEAU}

Anne Joyeau est Maître de conférences à l'IGR - IAE de l'Université de Rennes 1 et membre du CREM, UMR CNRS 6211. Spécialité disciplinaire : Gestion des ressources humaines.

Domaines de recherche : développement soutenable et responsabilité sociale des entreprises, normes sociales, commerce éthique. Mail : anne.joyeau@univ-rennes1.fr

\section{PHILIPPE ROBERT-DEMONTROND}

Mail : philippe.robert@univ-rennes1.fr 